\title{
Computational design of peptide therapeutics: how useful are sequence-based protein-protein interaction predictors?
}

\author{
François Charih ${ }^{1,2}$, Kyle K. Biggar ${ }^{2}$, James R. Green ${ }^{1 *}$ \\ 1 Department of Systems and Computer Engineering, Carleton University, Ottawa, \\ Ontario, Canada \\ 2 Institute of Biochemistry, Carleton University, Ottawa, Ontario, Canada \\ * jrgreen@sce.carleton.ca
}

\begin{abstract}
Engineering peptides to achieve a desired therapeutic effect through the inhibition of a specific target activity or protein interaction is a non-trivial task. Few of the existing in silico peptide design algorithms generate target-specific peptides. Instead, many methods produce peptides that achieve a desired effect through an unknown mechanism. In contrast with resource-intensive high-throughput experiments, in silico screening is a cost-effective alternative that can prune the space of candidates when engineering target-specific peptides. Using a set of FDA-approved peptides we curated specifically for this task, we assess the applicability of several sequence-based protein-protein interaction predictors as a screening tool within the context of peptide therapeutic engineering. We show that similarity-based protein-protein interaction predictors are more suitable for this purpose than the current state-of-the-art deep learning methods. We also show that this approach is mostly useful when designing new peptides against targets for which naturally-occurring interactors are already known, and that deploying it for de novo peptide engineering tasks may require gathering additional target-specific training data. Taken together, this work offers evidence that supports the use of similarity-based protein-protein interaction predictors for peptide therapeutic engineering, especially peptide analogs.
\end{abstract}

\section{Author summary}

Peptides are short chains of amino acids that represent an alternative to small molecule drugs to inhibit enzymatic activity or protein-protein interactions. Designing small peptides to target a protein specifically is challenging, though a number of computational approaches do exist. These methods must to evaluate the quality of a peptide in terms of its ability to bind specifically to a target protein, which can be done in silico with sequence-based protein-protein interaction predictors. It isn't clear, however, how suitable the different types of predictors are for the task of predicting interactions between peptides and proteins. Herein, we apply state-of-the-art protein-protein interaction predictors to a set of FDA-approved peptide therapeutics to determine how the different protein-protein interaction predictors compare for the task of assessing peptide quality, and to determine under which circumstances these methods may be successful. Our results show that similarity-based predictors outperform deep learning methods, and that these methods may be more suitable for peptide analog engineering than for the design of peptide therapeutics de novo. 


\section{Introduction}

\section{In silico screening for peptide therapeutic engineering}

Peptide therapeutics represent a unique opportunity to interfere with abnormal enzymatic activity or to disrupt protein-protein interactions (PPIs) in a targeted fashion. In contrast with small molecules, peptides can often be designed for very high specificity against their target, thereby conferring them an advantageous safety profile. Given that peptides are simply chains of amino acids, the chemical space they span is, for all intents and purposes, chemically accessible in its entirety.

In spite of their advantages, peptide therapeutics represent an exceedingly small fraction of all approved therapeutics, with only 70 compounds approved in the U.S., Europe and Japan as of 2020 [1]. In practice, oral delivery of peptide therapeutics remains a sizable challenge due to their low bioavailability caused by the presence of proteases in the gastrointestinal tract, and size and charge constraints associated with the permeability of the mucosal membrane of the gut, among others [2].

As a result, almost all peptides currently in clinical use must be delivered intravenously [3]. Nevertheless, there is a shared optimism that advances in targeted delivery technologies will significantly lower the barriers associated with oral peptide delivery [3]. For example, liposomal delivery of peptide therapeutics appears to be promising option for multiple routes of administration (oral, intranasal, pulmonary, etc.) [4]. Other technologies such as hydrogels and permeation enhancing molecules are also currently being investigated, though more work needs to be done before they can be brought to the clinic [3]. It is thus reasonably foreseeable that peptides may garner a renewed interest in the near future.

In silico peptide engineering for therapeutic applications is already an active area of research, with multiple groups having leveraged advances in deep learning to develop novel algorithms for the development of antimicrobial peptides [5-7], anti-cancer peptides [8], and anti-hypertensive peptides [9], among others. Many of these methods generate peptides expected to achieve a certain effect; however, their exact target is often unknown. Some of these methods leverage neural networks trained on large datasets to learn the distribution of peptides possessing the desired properties. Peptides can then be sampled from such distribution to generate novel peptides that are similar, but different to those in the training set $[5,8]$. Other peptide engineering methods evaluate the fitness of peptides in vitro (e.g. $\mathrm{IC}_{50}$ in $[6]$ ).

Surprisingly few target-specific peptide engineering approaches have been developed $[10,11]$. This is unsurprising, given that validated protein-peptide interactions are relatively scarce in comparison with protein-protein interactions. As such, it is difficult to develop general-purpose peptide engineering algorithms that could be broadly applied to design a peptide that interacts with one's protein target of choice. To the best of our knowledge, InSiPS [11] is the only entirely in silico method that addresses this problem as of today.

Regardless of the peptide engineering approach used, the ability to identify a therapeutic peptide's target or to evaluate the likelihood that it will interact specifically with a target protein is highly desirable. Such a method would provide a quick, cheap way to identify candidates in preparation for more time-consuming and expensive in vitro or in vivo validation experiments.

\section{Sequence-based protein-protein interaction predictors}

Several sequence-based PPI prediction algorithms can evaluate the likelihood of an interaction between proteins. However, no widely used method has been developed specifically for the prediction of protein-peptide interactions. In theory, nothing 
precludes traditional PPI predictors from making predictions for pairs involving a short peptide, but in practice many of these predictors are trained on pairs involving large proteins. As such, it is not yet clear how applicable these methods are for protein-peptide interactions.

Though generally thought to provide less accurate predictions than structure-based methods, sequence-based predictors are widely used and appreciated because they can generate predictions for any pair of proteins, so as long as an amino acid sequence is available; however, it should be mentioned that several predictors do require proteins to have a minimum length. While protein-peptide docking simulations can provide a wealth of thermodynamic information that sequence-based predictors cannot, few proteins are amenable to these experiments. In general, the resolution of protein

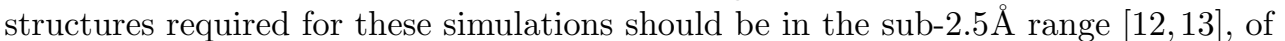
which there are relatively few, though reasonably accurate structures predicted with AlphaFold 2 [14] are now available for most proteins. Furthermore, sequence-based predictors tend to require far fewer computational resources to run compared with structure-based methods which may require iterative docking, a resource-intensive procedure. In fact, our massively parallel implementation of the SPRINT algorithm [15] can predict the entire human interactome in under an hour using a 40-core machine with 64 GB memory. As such, sequence-based PPI predictors provide a unique opportunity to quickly and inexpensively screen synthetically designed peptides and to assess their potential as candidates in wet lab experiments.

Two broad types of approaches are currently being used to predict protein-protein interactions: similarity-based methods and machine learning-based methods (Figure 1). Similarity-based methods such as PIPE4 [16] and SPRINT [15] score proteins based on the fundamental idea that a pair of known interacting proteins $P 1$ and $P 2$ provides evidence for an interaction between the query proteins $Q 1$ and $Q 2$ if $P 1$ is similar to $Q 1$ and $P 2$ is similar to $Q 2$. These methods, in essence, quantify the strength of the evidence for an interaction under this assumption, using substitution matrices such as PAM120 or BLOSUM64 to assess the similarity between query and interacting protein pairs. These methods are close to what one may call instance-based learning in machine learning terminology. In contrast, machine learning-based methods "learn" to recognize patterns or features that occur frequently in interacting proteins. To make predictions, the machine learning models then look for the presence or extent of these patterns in query protein pairs. Depending on the authors, the predictors may learn patterns from the physicochemical properties of the protein sequences or simply from the amino acid composition (eg. di- and tri-peptide composition, pseudo-amino acid composition, etc.). The most recent models tend to implement the latter approach with powerful deep learning models to learn the "grammar" of interacting proteins [17-19].

The PIPE sequence-based PPI predictor [16] has been successfully used to design novel peptides that interact with a selected target. The In Silico Peptide Synthesizer (InSiPS) [11], built around PIPE, uses genetic algorithms to explore the peptide space and maximize peptide fitness. The fitness function is designed such that the predicted interaction score between the peptide and the target is maximized, but the interaction score between the peptide and all other proteins is minimized. This ensures that the algorithm favours peptides that are predicted to interact specifically with the protein target. So far, InSiPS has been demonstrated to produce valid peptides, but no published work has shown that it can produce valid peptide binding to human targets. Furthermore, little work has been done to validate the use of recent deep learning PPI prediction models for applications in peptide engineering. 


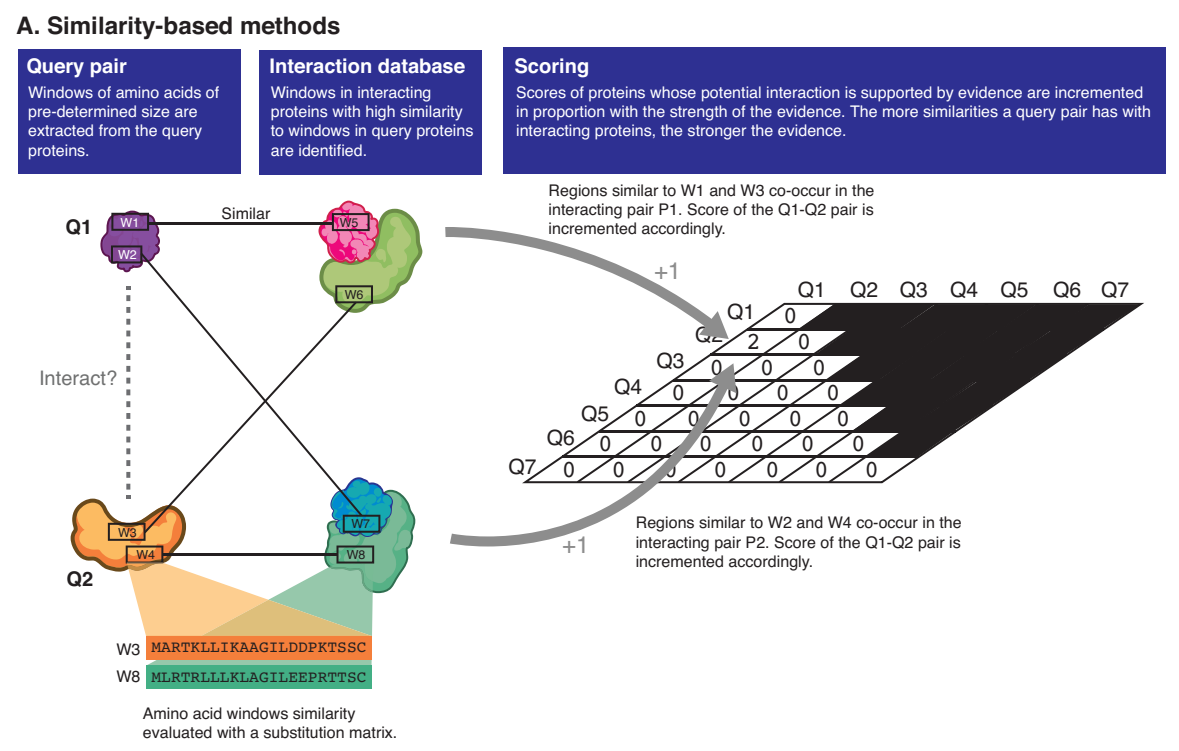

B. Machine learning-based methods
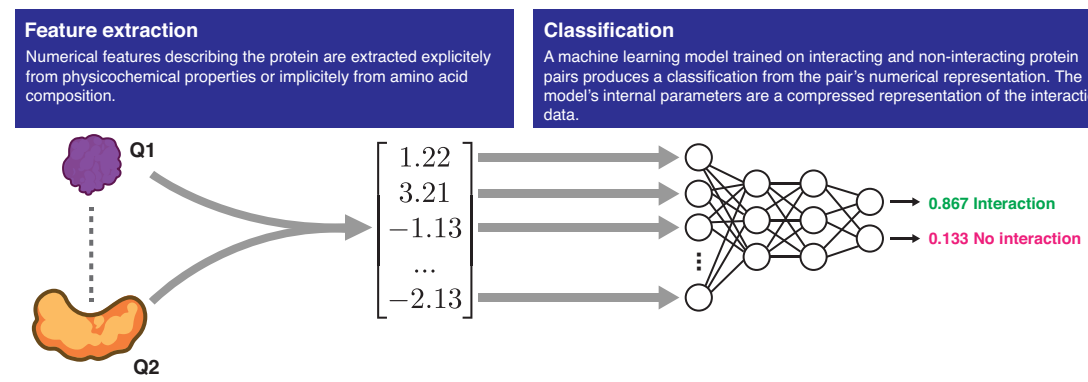

Fig 1. Similarity- and machine learning-based protein-protein interaction predictors. Similarity-based methods differ from machine learning-based methods in that the evidence supporting an interaction is quantified by counting the number of similarities to known instances of interacting proteins in a database. Machine learning-based methods are trained to learn patterns found in interacting protein pairs, and identify those patterns in the query proteins.

\section{The one-to-all curve for a qualitative assessment of peptide quality}

The one-to-all curve is a simple, yet informative visualization of the interaction landscape of a protein-binding compound. Information contained within the curve has been shown to be useful in the context of PPI [20] and miRNA target prediction tasks [21]. It is obtained by plotting the predicted interaction score of all proteins expressed in the target organism, tissue, or subcellular location as a function of the ranking of that score, relative to the score of other proteins, for one peptide. The morphology of the curve enables a qualitative assessment of a peptide in terms of its ability to bind the intended target and its specificity.

Within the context of peptide-protein interactions, most one-to-all curves for peptides are L-shaped or sigmoidal. In the more common L-shaped curve, most proteins lie along a baseline representing the proteins to which a very low interaction score with the peptide is predicted, indicating that an interaction is not supported by the evidence (training data) used to train the model. In the context of peptide engineering, all but 
very few peptides will produce a curve where the targeted protein lies along the baseline (Figure 2A), consistent with the idea that very few peptides will actually bind to a given protein target.

(A) No interaction

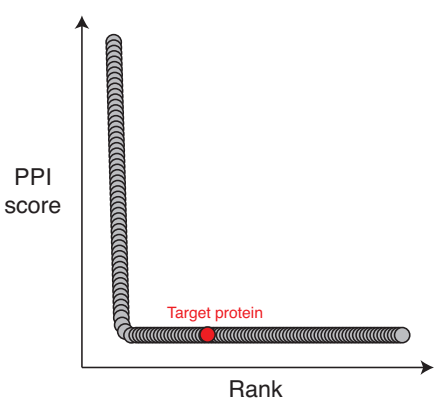

(B) Non-specific interaction

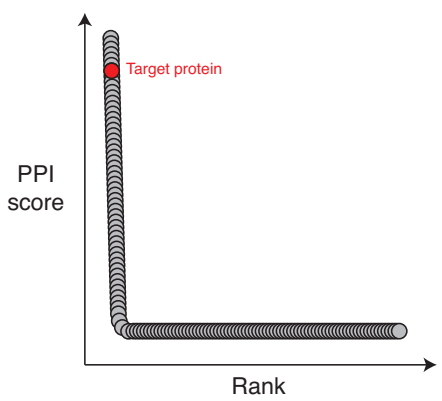

(C) Ideal curve

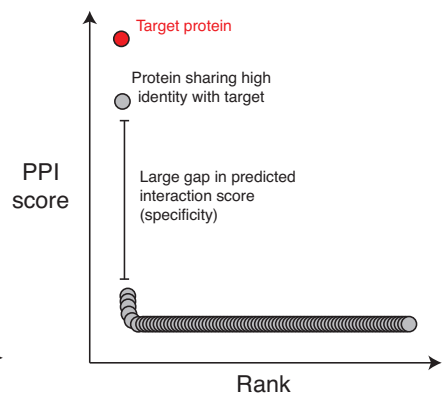

Fig 2. Representative one-to-all curves. Many one-to-all curves adopt one of three morphologies: (A) no interaction between the peptide and the target, (B) non-specific interaction where the peptide is predicted to interact with many proteins in addition to the target, and (C) where the peptide specifically interacts with the intended protein target (and possibly, closely related proteins).

The number of proteins which lie above the elbow of the curve provide information with respect to the specificity of the peptide, i.e. whether the peptide is predicted to interact with multiple proteins. As such, it is not only desirable for the target protein to be as high above the elbow as possible, as it must be one of the few high-scoring proteins, it must also be higher scoring than other non-target proteins. In many cases, a highly non-specific interaction with a protein target will be predicted, yielding a curve such as the one shown in Figure 2B. Such peptides are unlikely to be useful in practice, due to the high likelihood of off-target interactions, potentially leading to undesirable side effects.

Figure $2 \mathrm{C}$ represents the most desirable scenario for a good peptide therapeutic candidate. The target protein is strongly predicted to interact with the target protein, and the only other proteins achieving a similar score are proteins with high similarity to the target (isoforms, for example). In such cases, peptide specificity may be further optimized in vitro.

The $y$ axis of the one-to-all curve is not always easy to interpret, particularly when the PPI prediction score is unbounded. Most machine learning-based predictors convert raw prediction scores into probabilities between 0 and 1 using the softmax function. However, similarity-based methods instead output a number that reflects the strength of the evidence, as is the case with SPRINT [15]. In such cases, it is the user's responsibility to identify an appropriate threshold value on the score above which protein pairs are predicted to interact. This can be done using cross-validation experiments.

\section{Contributions}

Herein, we evaluate the applicability of sequence-based PPI predictors in identifying peptides fit for in vitro or in vivo validation. Using a set of FDA-approved peptides, we compare three state-of-the-art sequence-based PPI predictors and their usefulness in evaluating peptides for creation of peptide analogous to endogenously expressed proteins, and for de novo peptide design for novel protein targets. We believe that this work has significant implications for peptide engineering and could guide use of PPI predictors in future peptide engineering pipelines. 


\section{Methods}

The code and instructions needed to reproduce the data and analyses presented here are available in the GitHub repository: https://github.com/GreenCUBIC/PeptideScreening.

\section{Assumption}

Several peptides have been approved by the FDA for the treatment of a number of conditions such as heart failure and diabetes. Given that these peptides have been shown to be safe, one could deduce that these peptides interact specifically with their target, i.e. interact with few proteins other than their intended target. Off-target interactions are unlikely to be numerous, as this would likely cause unintended side effects.

This assumption underpins the interpretation of one-to-all curves generated as part of this work. In other words, FDA-approved peptides should, in theory, rank their known target protein among the first proteins on the one-to-all curve and score much higher than other proteins.

\section{Curating FDA-approved peptides}

To identify suitable peptide therapeutics for assessment with PPI predictors, we retrieved a list of all therapeutic peptides approved by the FDA for therapeutic use as of 2017, as compiled previously [22]. Of those 69 peptides, we selected those whose length was greater than 20 amino acids and only comprised standard amino acids (see Table 1), leaving a total of 13 peptides. This additional round of curation was necessary, because certain predictors cannot predict interactions involving very small protein fragments and/or non-standard amino acids. Finally, for each peptide, we retrieved the associated protein target(s) from the DrugBank database [23].

Table 1. FDA-approved peptides evaluated in this study

\begin{tabular}{|c|c|c|c|c|}
\hline Compound Name & Molecular Target & Chemical Basis & Length & Known Targets (Accession ID) \\
\hline Corticotropin & $\mathrm{MC}$ receptors & native & 39 & Q01726;Q01718;P41968;P32245;P33032 \\
\hline Calcitonin (salmon) & $\begin{array}{l}\text { Calcitonin } \\
\text { receptor }\end{array}$ & native & 32 & P30988 \\
\hline Tetracosactide & $\mathrm{MC}$ receptors & native & 24 & Q01726;Q01718;P41968;P32245;P33032 \\
\hline Calcitonin (human) & $\begin{array}{l}\text { Calcitonin } \\
\text { receptor }\end{array}$ & native & 32 & Q16602 \\
\hline Carperitide & NPR-A & native & 28 & P16066;P20594;P17342 \\
\hline Bivalirudin & Thrombin & analog & 20 & P00734 \\
\hline Nesiritide & NPR-A & native & 32 & $\mathrm{P} 16066 ; \mathrm{P} 20594 ; \mathrm{P} 17342$ \\
\hline Pramlintide & $\begin{array}{l}\text { Calcitonin } \\
\text { receptor }\end{array}$ & analog & 37 & Q16602 \\
\hline Exenatide & GLP-1 receptor & native & 39 & P43220 \\
\hline Liraglutide & GLP-1 receptor & analog & 32 & P43220 \\
\hline Tesamorelin & GHRH receptor & analog & 44 & Q02643 \\
\hline Teduglutide & GLP-2 receptor & analog & 33 & O95838 \\
\hline Lixisenatide & GLP-1 receptor & analog & 44 & P43220 \\
\hline
\end{tabular}

148 149

\section{0}

151

152

153

154

155

156

157

158

159

160

161

162

163

164

165

166 


\section{Training and deploying PPI predictors to score interactions}

We selected 3 state-of-the-art sequence-based PPI predictors for comparison (Table 2). Two of them (PIPR and D-SCRIPT) are deep learning-based, whereas the other (SPRINT) is similarity-based.

Both PIPR and D-SCRIPT generate protein embeddings to use as inputs to deep neural networks. In other words, the protein sequences are projected into a latent space, where the coordinates relative to those of other protein pairs capture their relationship in terms of their sequence similarity or distance. There are a number of ways one may generate embeddings. The authors of PIPR [18], for instance, concatenate the sub-embeddings obtained from residue co-occurence obtained by training a Skip-Gram model with another sub-embedding obtained by clustering amino acids into 7 classes of electrostaticity and hydrophobicity to generate a one-hot encoding (second

sub-embedding). On the other hand, D-SCRIPT [24] uses the existing Bepler \& Berger embedding [25], which is generated with a bidirectional long short-term memory neural network. The embedding generator is trained using three tasks involving two input proteins: prediction of the shared SCOP level of the proteins, self-contact maps of their 3D structures and the "soft symmetric alignment".

While a D-SCRIPT model pre-trained on human PPIs is available, both PIPR and SPRINT require validated interaction data to make predictions. PIPR, like any machine learning-based binary classifier, requires positive and negative protein pairs for training. In contrast, SPRINT only takes interacting protein pairs (positive examples) as an input. These interactions are not used to "train" SPRINT per se, as SPRINT is not a machine learning method in the conventional sense, but are instead used as a database which the algorithm queries against in search of similarities with a query protein pair.

To generate the training set for PIPR and generate the SPRINT database, we retrieved all PPIs involving two human proteins from the BioGRID database (version 4.3.196) [26] and filtered them so as to only retain high-quality interactions [27], i.e. interactions reported by at least two groups, and detected with stringent experimental methods [27]. We added pairs assumed to be non-interacting to PIPR's training set by adding random protein pairs to produce a balanced training set. We then trained PIPR for 100 epochs on this training set and generated the one-to-all curves for all 13 peptides. Similarly, we produced the one-to-all curves using SPRINT and the pre-trained D-SCRIPT model.

Given that all but one selected peptides target receptors expressed on the cell surface, all one-to-all curves were generated using proteins predicted to be exposed on the cell's surface as opposed to the entire proteome. We obtained the list of the 2,886 proteins predicted to make up the human surfaceome from The in silico surfaceome [28]. We added thrombin, a protein involved in the coagulation cascade to all curves, because it is targeted by one of the therapeutic peptides (bivalirudin).

\section{Simulating peptide screening in de novo engineering}

Given that all peptides under study are actually truncated segments of endogenously expressed proteins ("native" in Table 1) or recombinant analogs, training the models using the endogenous equivalents of the therapeutic peptides represents an optimistic scenario. In that "optimistic" scenario, we know that an interaction highly similar or identical to that we are attempting to predict is present in the training data. This mimics a scenario where we wish to design a peptide with some similarity to an endogenously expressed protein known to interact with the protein target.

For this reason, we ran blastp [29] with the default parameters to identify proteins which share a region of high similarity with the therapeutic peptides. All interactions involving hits found via the BLAST searched were removed from our PPI dataset to 
produce training data for a "pessimistic" scenario representing the de novo case. This pessimistic scenario, denoted (-) as opposed to the optimistic scenario (+), reflects what one may encounter when designing peptides against a target for which no interactors are known.

After re-training PIPR on the pessimistic dataset, we re-generated the one-to-all curves using this pessimistic PIPR model and SPRINT.
Table 2. State of the art sequence-based PPI predictors

\begin{tabular}{|c|c|c|c|c|c|}
\hline Predictor & Type & $\begin{array}{l}\text { Machine learn- } \\
\text { ing model }\end{array}$ & Features & Availability & Ref. \\
\hline SPRINT & Similarity-based scoring & Not applicable & Not applicable & $\mathrm{C}++$ source & [15] \\
\hline PIPR & Machine learning & $\mathrm{RCNN}$ & $\begin{array}{l}\text { Latent representation of pro- } \\
\text { tein sequences generated by } \\
\text { a combination of convolution } \\
\text { layers and re- sidual gated } \\
\text { recurrent units }\end{array}$ & Python source & [18] \\
\hline D-SCRIPT & Machine learning & Bi-LSTM + CNN & $\begin{array}{l}\text { Combination of a pre-trained } \\
\text { embedding model with a pre- } \\
\text { dicted contact map }\end{array}$ & $\begin{array}{l}\text { Python pack- } \\
\text { age }\end{array}$ & [24] \\
\hline
\end{tabular}

\section{Results}

\section{SPRINT outperforms deep learning models}

The rank of the targeted protein(s) along the one-to-all curve conveys a lot of information with respect to the potential of a peptide as a therapeutic, since it is desirable to optimize for specific interactions. In general, a peptide whose target protein is outranked by hundreds or thousands of off-target proteins on the one-to-all curve is very unlikely to make a good candidate, under the assumption that the predictions are accurate.

With the aforementioned assumption in mind, we found that D-SCRIPT did not assign high ranks to the known targets on the one-to-all curves, but also failted to predict an interaction for any of the peptide-target pairs, assigning an interaction probability close to 0 in each case. The median rank of the peptide-target pairs on the respective one-to-all curves is $1,330 / 2,886$, with only one peptide-target pair ranking in the top 500 on its corresponding one-to-all curve, and none ranking in the top $1 \%$ (Figure 3B).

PIPR performed better than D-SCRIPT, for both scenarios considered. In the pessimistic scenario analogous to assessment of de novo peptides, PIPR predicted an interaction (interaction probability $>50 \%$ ) for 3 out of the 21 peptide-target pairs, though no peptide-target pairs ranked in the top $1 \%$ on their one-to-all curve. In the optimistic scenario, however, where interactions involving proteins with high similarity to the therapeutic peptides are included in the training interactions, PIPR ranks five peptide-target pairs in the top $1 \%$ of their respective one-to-all curve. While PIPR did rank targets more highly than non-targets in more cases than D-SCRIPT, it should be noted that it produced a number of curves with a sigmoidal morphology with many high-scoring proteins (see Supporting Information). This suggests that PIPR may be particularly prone to false positives.

SPRINT is the predictor that achieved the highest success, scoring six true targets among the top 1\% proteins on their curve in the pessimistic scenario, and 16 in the optimistic scenario. The median rank of the peptide-target pairs is also predicted to be 
lower by SPRINT than by PIPR in both scenarios. In general, it appears that SPRINT outperforms deep learning predictors for the task of predicting protein-peptide interactions, particularly in the optimistic scenario where all known interactions are leveraged to make predictions.
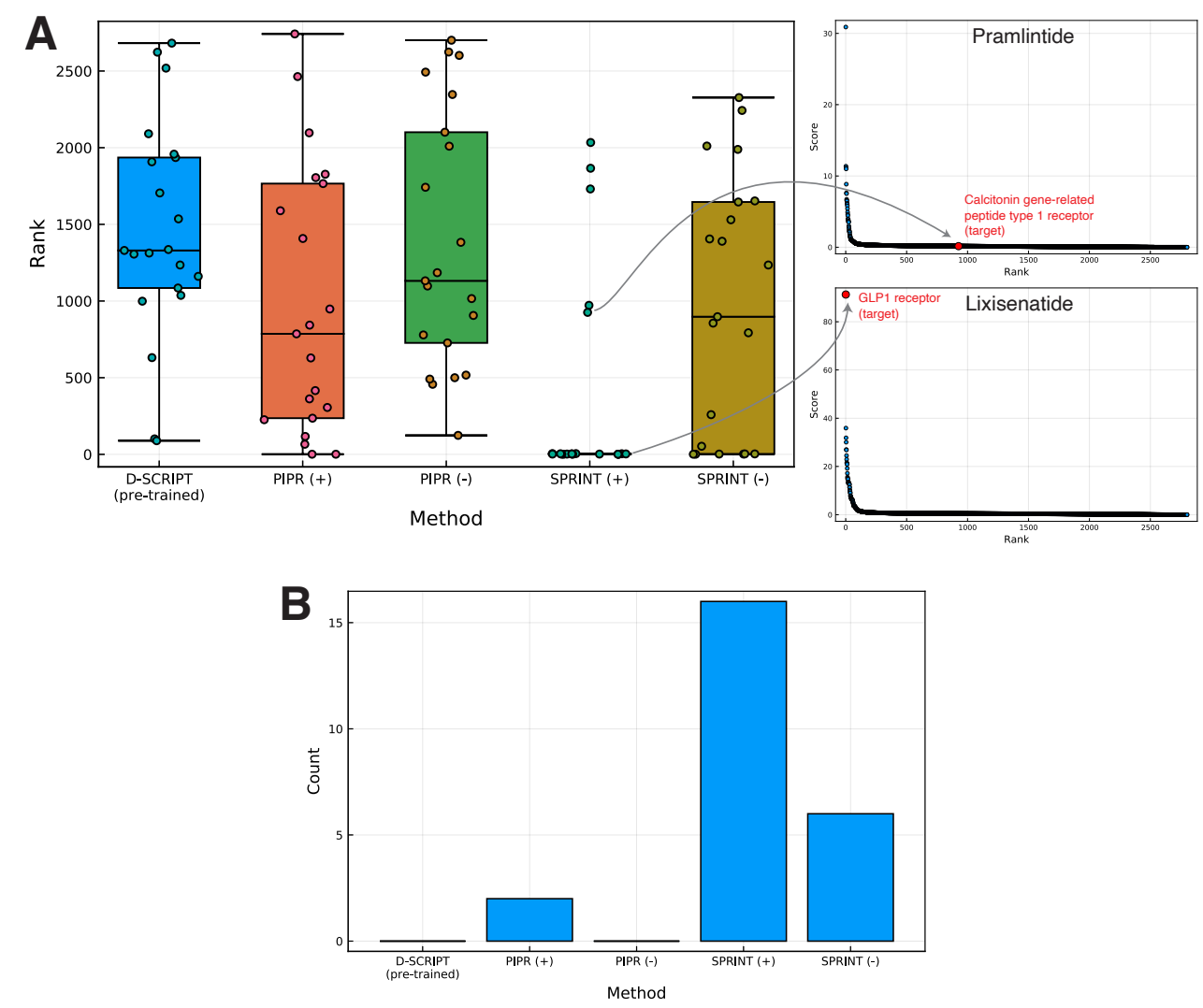

Fig 3. Rankings of interactions involving peptide-target pairs (A)

Distributions of ranks of the peptide-target pairs are shown for models whose training data included interactions involving an endogenous peptide analog (optimistic scenario; + ) and models trained without (pessimistic scenario; -). The ranks of the true targets for two therapeutic peptides are illustrated on their one-to-all curves on the right side of the box plot. (B) Counts of therapeutic peptide targets ranking in the top $1 \%$ on the corresponding one-to-all curve.

\section{Protein-protein interaction predictors are useful for peptide analog screening}

Consistent with the results above, screening for peptide analogs with some similarity to endogenously expressed proteins is a scenario where SPRINT outperforms the other predictors, as illustrated with lixisenatide (in Figure 4A).

Lixisenatide is a 44 amino acids glucagon-like peptide- 1 agonist marketed by Sanofi and used in the treatment of type 2 diabetes to increase insulin secretion. This peptide is an GLP-1 analog corresponding to a portion of the exendin-4 protein found in the venom of Gila monster (Heloderma suspectum) [30]. The target, the glucagon-like peptide 1 receptor (Accession ID: P43220), is a G-protein coupled receptor located on the cell surface. As such, we included only surface proteins in the one-to-all curves 
(Figure 4).

The one-to-all curves show that both D-SCRIPT and PIPR assign low interaction probabilities between lixisenatide and the GLP1 receptor. The target proteins ranking poorly along the curves. In fact, the probability being well below 0.5 and no interactions are predicted to occur. In the case of D-SCRIPT, 16 proteins are predicted to interact with lixisenatide, none of them being the GLP1 receptor. In fact, D-SCRIPT predicts a $0.4 \%$ chance of an interaction between lixisenatide and the GLP1 receptor. PIPR predicts an interaction with higher probability, though it still fails to meet the 0.5 threshold while assigning higher scores to over 500 non-target proteins on the cell's surface in both the optimistic and pessimistic scenarios. SPRINT, when "trained" in the optimistic scenario, accurately ranks the GLP1 receptor as first on the one-to-all curve while assigning much lower scores to other off-target proteins.
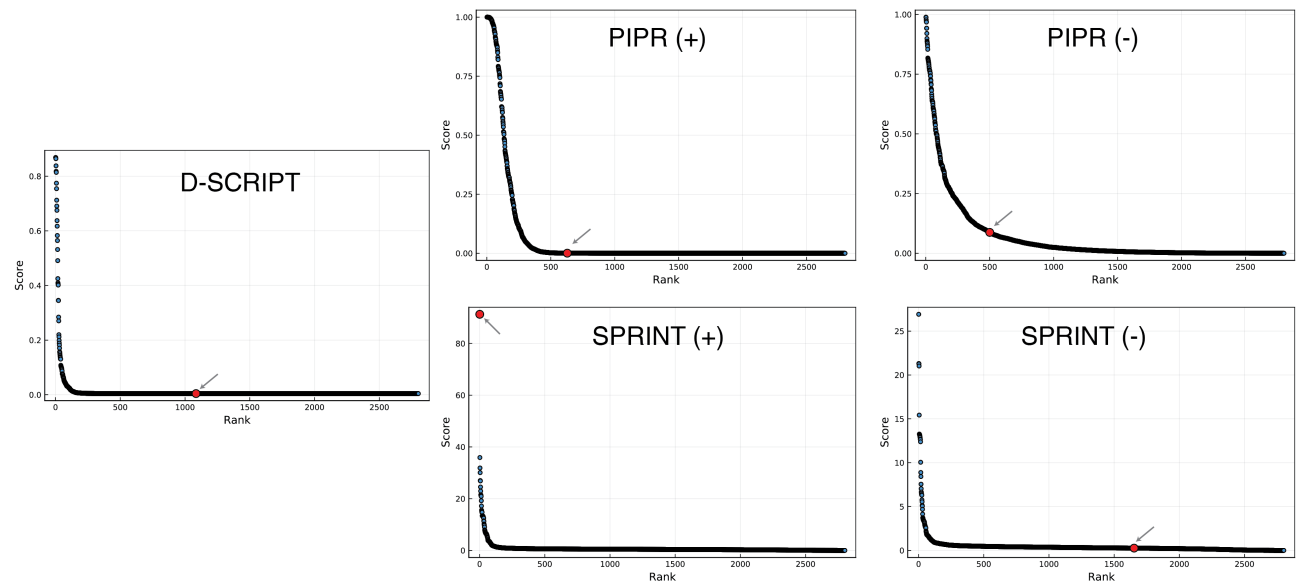

Fig 4. One-to-all curves for lixisenatide. The one-to-all curves generated using the predicted interaction scores of the three predictors are shown here. The true target protein is highlighted in red, while the black dots represent off-target proteins. One-to-all curves generated with predictors trained in the optimistic scenario are indicated with a $(+)$.

Only SPRINT makes credible predictions under the assumption that lixisenatide interacts specifically with the GLP1 receptor, and only in the optimistic case, i.e. when the interaction between (pro)-glucagon (Accession ID: P01275) and the GLP1 receptor is included in the training data. In fact, alignment shows that lixisenatide, though not derived from human glucagon, aligns to a certain degree with a portion of it (98-141), part of which (98-128) is normally cleaved to yield the GLP1 peptide Figure 5. This suggests that SPRINT can effectively be leveraged to screen peptide analogs that are somewhat similar, but not identical to endogenously expressed proteins.

\section{Assessing peptide fitness for de novo design remains challenging}

All predictors, including SPRINT, generally failed to rank targets highly on the one-to-all curve in the more challenging de novo screening scenario, i.e. where interactions involving proteins with high similarity with to the therapeutic peptides were excluded from the training data.

In a limited number of cases, SPRINT assigns better ranks to peptides in the pessimistic scenario. Such was the case for carperitide, a peptide marketed by Daiichi Sankyo for the treatment of acute heart failure. Both in the presence and absence of training interactions involving the $\alpha$-atrial natriuretic peptide, of which carperitide is a 


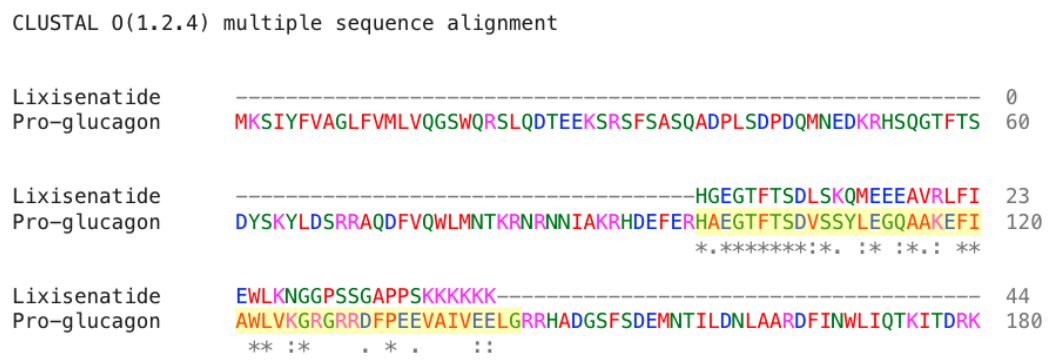

Fig 5. Alignment between lixisenatide and pro-glucagon. Results of the alignment between lixisenatide and pro-glucagon generated with Clustal Omega [31]. The highlighted region corresponds to the endogenously expressed GLP1 peptide.

recombinant version, SPRINT ranked the intended target highly on the one-to-all curves (Figure 6), albeit with a lower score than in the optimistic scenario $(+)$. In these curves, all three best-ranked proteins are isoforms of the atrial natriuretic peptide receptor. We observed similar results for nesiritide, which is also a recombinant version of a natriuretic peptide.

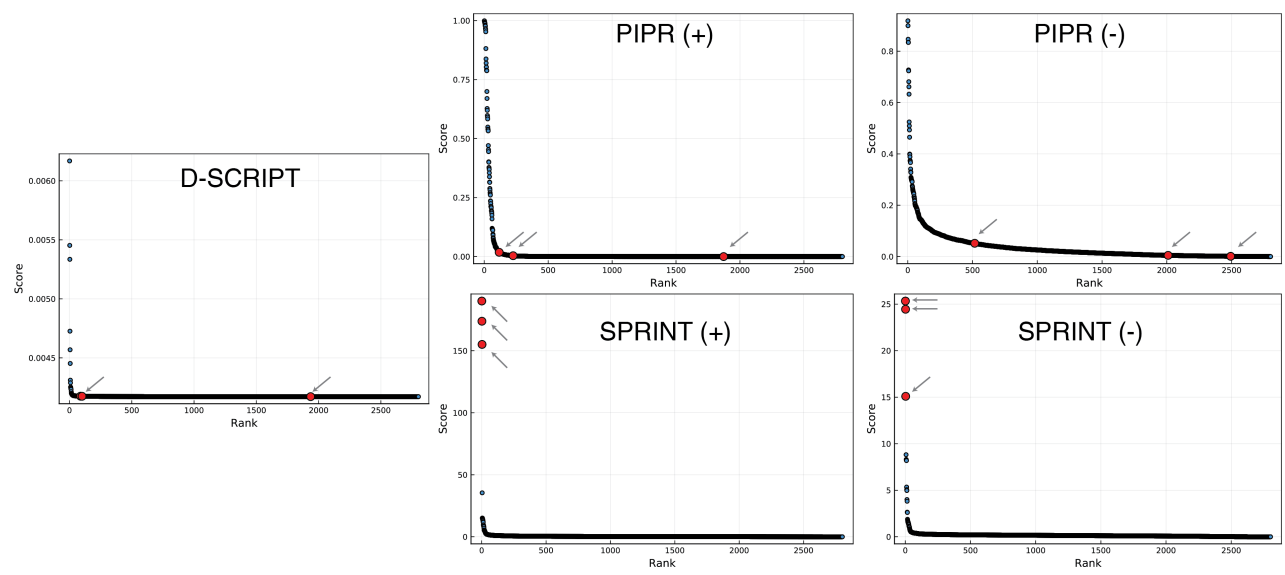

Fig 6. One-to-all curves for carperitide. The one-to-all curves generated using the predicted interaction scores of the three predictors are shown here. The true target protein is highlighted in red, while the black dots represent off-target proteins. One-to-all curves generated with predictors trained in the optimistic scenario are indicated with a $(+)$.

\section{Discussion}

The ability to rapidly and inexpensively screen peptides in silico as potential therapeutics targeting a specific protein is highly desirable, but remains challenging. This can be partially attributed to the relative scarcity of validated peptide-protein interactions. Given that most PPI predictors are trained using long proteins, we anticipated that these methods would perform poorly when deployed for the task of predicting interactions involving shorter peptides ( $<50$ amino acids). 


\section{The case for similarity-based methods}

D-SCRIPT and PIPR, both deep learning methods, mostly failed to produce realistic one-to-all curves. However, SPRINT produced reasonably good one-to-all curves that ranked known targets better than non-targets, particularly when all available protein-protein interactions were used to make predictions. This suggests that SPRINT may be suitable as a tool to screen peptides in cases where an endogenous interactor is known for the target protein. Deep learning methods, in contrast, are generally not. We reason that SPRINT outperforms the competing methods because of its prediction mechanism. Deep learning methods make predictions based on both the global and local properties of protein sequences which are captured in an embedding. This is in contrast with SPRINT, whose predictions rely on small, local protein regions of similarity that are roughly the length of a peptide ( $\sim 20$ amino acids). The fact SPRINT uses what essentially amounts to short peptides as evidence to support their predictions makes SPRINT and other similarity-based PPI predictors (eg. PIPE [16]) uniquely suited for such tasks.

\section{Sequence-based methods achieve limited success in de novo design tasks}

Unsurprisingly, we found that assessing the quality of peptides that have little to no high-similarity regions to proteins in the training dataset of interacting proteins is challenging. In fact, SPRINT is sensitive to small changes in the training data, as top-ranking peptide-target pairs often dropped to the bottom of the one-to-all curve when one or two key interactions were removed from the training data (pessimistic scenario). For this reason, applying SPRINT to screen de novo peptides for potential interactions with a target is likely to be unsuccessful, depending on the training data. However, SPRINT assigned high ranks to the target (natriuretic peptide receptor A) for both carperitide and nesiritide, even in the absence of their endogenous equivalent in the training data under the pessimistic scenario. Our data do not support the generalization of this result to other peptides, especially considering that carperitide and nesiritide target the same receptor.

\section{Rank on the one-to-all curve is a key metric}

SPRINT produces an unbounded sum of the regions of similarity between query proteins and proteins in the training set of interacting proteins, weighted by the similarity as scored with a substitution matrix. That raw sum corresponds to the prediction score, and the threshold separating non-interacting pairs from interacting pairs is arbitrary. Normally, a reasonable threshold could be determined through cross-validation for normal protein-protein interaction tasks. We believe the rank along the one-to-all curve to be just important than the raw score of a target, especially in the context of peptide engineering. A low predicted interaction score may be due to limited similarity to regions in known interacting proteins. However, for peptide engineering tasks, such a cross-validation experiment is not possible, since no a novel peptide would have no known and validated interactions. Thus it may be worth validating a high-ranking peptide in vitro, even if the raw interaction score is low. More work will be needed to develop a peptide fitness metric that incorporates both the rank of the target protein along the one-to-all curve, and the predicted score and other features derivable from the curve. 


\section{Tackling the challenges associated with de novo peptide design}

While general-purpose methods like SPRINT show some potential in a purely in silico context for design of peptide analogs, we believe that incorporating target-specific training data obtained experimentally could increase the applicability of similarity-based PPI prediction methods for de novo peptide engineering tasks. For example, sparsely sampling the peptide space ( 1,000 peptides) and testing for interactions with the target protein using peptide arrays could supplement the initial training data and drastically improve the predictions. In fact, one group found that sparse sampling of the peptide space for target-specific peptides could provide the training data necessary to train a simple neural network that could predict with reasonable accuracy whether a peptide would bind to that specific target [32]. Using predictors like SPRINT in an adaptive fashion could also significantly enhance their applicability in peptide engineering task. For instance, peptides optimized in silico could periodically be tested experimentally for binding to the target. Hits could then be incorporated in the training data in an iterative fashion and improve the quality of the predictions in future iterations.

\section{Concluding remarks}

In summary, our work shows that similarity-based PPI predictors are currently more suitable than deep learning methods to evaluate the potential of peptide therapeutics. We also showed that deploying such methods for analog peptide engineering is more likely to be successful than for engineering peptides de novo, i.e. against targets for which no interactors are known. We anticipate that more specialized protein-peptide interaction predictors will become available as more data becomes available, but believe that existing methods can already be integrated in peptide engineering pipelines. Finally, we believe that adaptive use of existing sequence-based PPI prediction methods holds great potential.

\section{Supporting information}

S1 Fig. All one-to-all curves generated with D-SCRIPT.

S2 Fig. All one-to-all curves generated with PIPR (optimistic scenario).

S3 Fig. All one-to-all curves generated with PIPR (pessimistic scenario).

S4 Fig. All one-to-all curves generated with SPRINT (optimistic scenario).

S5 Fig. All one-to-all curves generated with SPRINT (pessimistic scenario).

\section{References}

1. Zhang Y, Zhang H, Ghosh D, Williams RO. Just How Prevalent are Peptide Therapeutic Products? A Critical Review. International Journal of Pharmaceutics. 2020;587:119491. doi:10.1016/j.ijpharm.2020.119491.

2. Drucker DJ. Advances in oral peptide therapeutics. Nature Reviews Drug Discovery. 2020;19(4):277-289. doi:10.1038/s41573-019-0053-0. 
3. Muttenthaler M, King GF, Adams DJ, Alewood PF. Trends in peptide drug discovery. Nature Reviews Drug Discovery. 2021;20(4):309-325. doi:10.1038/s41573-020-00135-8.

4. Swaminathan J, Ehrhardt C. Liposomal delivery of proteins and peptides. Expert Opinion on Drug Delivery. 2012;9(12):1489-1503. doi:10.1517/17425247.2012.735658.

5. Müller AT, Hiss JA, Schneider G. Recurrent Neural Network Model for Constructive Peptide Design. Journal of Chemical Information and Modeling. 2018;58(2):472-479. doi:10.1021/acs.jcim.7b00414.

6. Yoshida M, Hinkley T, Tsuda S, Abul-Haija YM, McBurney RT, Kulikov V, et al. Using Evolutionary Algorithms and Machine Learning to Explore Sequence Space for the Discovery of Antimicrobial Peptides. Chem. 2018;4(3):533-543. doi:10.1016/j.chempr.2018.01.005.

7. Lin Y, Cai Y, Liu J, Lin C, Liu X. An advanced approach to identify antimicrobial peptides and their function types for penaeus through machine learning strategies. BMC Bioinformatics. 2019;20(Suppl 8):291. doi:10.1186/s12859-019-2766-9.

8. Grisoni F, Neuhaus CS, Gabernet G, Müller AT, Hiss JA, Schneider G. Designing Anticancer Peptides by Constructive Machine Learning. ChemMedChem. 2018;13(13):1300-1302. doi:10.1002/cmdc.201800204.

9. Manavalan B, Basith S, Shin TH, Wei L, Lee G. mAHTPred: a sequence-based meta-predictor for improving the prediction of anti-hypertensive peptides using effective feature representation. Bioinformatics. 2018;35(16):2757-2765. doi:10.1093/bioinformatics/bty1047.

10. Tallorin L, Wang J, Kim WE, Sahu S, Kosa NM, Yang P, et al. Discovering de novo peptide substrates for enzymes using machine learning. Nature Communications. 2018;9(1):5253. doi:10.1038/s41467-018-07717-6.

11. Burnside D, Schoenrock A, Moteshareie H, Hooshyar M, Basra P, Hajikarimlou $\mathrm{M}$, et al. In silico engineering of synthetic binding proteins from random amino acid sequences. iScience. 2018;11:375-387. doi:10.1016/j.isci.2018.11.038.

12. Brylinski M, Skolnick J. Q [U+2010] Dock: Low [U+2010] resolution flexible ligand docking with pocket [U+2010] specific threading restraints. Journal of Computational Chemistry. 2008;29(10):1574-1588. doi:10.1002/jcc.20917.

13. Kinghorn AD, Falk H, Gibbons S, Kobayashi J, Asakawa Y, Liu JK. Progress in the Chemistry of Organic Natural Products 110: Cheminformatics in Natural Product Research. Progress in the Chemistry of Organic Natural Products. Springer International Publishing; 2019. Available from: https://books.google.ca/books?id=aiK3DwAAQBAJ.

14. Jumper J, Evans R, Pritzel A, Green T, Figurnov M, Ronneberger O, et al. Highly accurate protein structure prediction with AlphaFold. Nature. 2021; p. 1-11. doi:10.1038/s41586-021-03819-2.

15. Li Y, Ilie L. SPRINT: ultrafast protein-protein interaction prediction of the entire human interactome. BMC Bioinformatics. 2017;18(1):485. doi:10.1186/s12859-017-1871-x. 
16. Dick K, Samanfar B, Barnes B, Cober ER, Mimee B, Tan LH, et al. PIPE4: Fast PPI Predictor for Comprehensive Inter- and Cross-Species Interactomes. Scientific reports. 2020;10(1):1390. doi:10.1038/s41598-019-56895-w.

17. Yao Y, Du X, Diao Y, Zhu H. An integration of deep learning with feature embedding for protein-protein interaction prediction. PeerJ. 2019;7:e7126. doi:10.7717/peerj.7126.

18. Chen M, Ju CJT, Zhou G, Chen X, Zhang T, Chang KW, et al. Multifaceted protein-protein interaction prediction based on Siamese residual RCNN. Bioinformatics. 2019;35(14):i305-i314. doi:10.1093/bioinformatics/btz328.

19. Hashemifar S, Neyshabur B, Khan AA, Xu J. Predicting protein-protein interactions through sequence-based deep learning. Bioinformatics. 2018;34(17):i802-i810. doi:10.1093/bioinformatics/bty573.

20. Dick K, Green JR. Reciprocal Perspective for Improved Protein-Protein Interaction Prediction. Scientific Reports. 2018;8(1):11694. doi:10.1038/s41598-018-30044-1.

21. Kyrollos DG, Reid B, Dick K, Green JR. RPmirDIP: Reciprocal Perspective improves miRNA targeting prediction. Scientific Reports. 2020;10(1):11770. doi:10.1038/s41598-020-68251-4.

22. Lau JL, Dunn MK. Therapeutic peptides: Historical perspectives, current development trends, and future directions. Bioorganic \& Medicinal Chemistry. 2018;26(10):2700-2707. doi:10.1016/j.bmc.2017.06.052.

23. Wishart DS, Feunang YD, Guo AC, Lo EJ, Marcu A, Grant JR, et al. DrugBank 5.0: a major update to the DrugBank database for 2018. Nucleic Acids Research. 2017;46(D1):gkx1037-. doi:10.1093/nar/gkx1037.

24. Sledzieski S, Singh R, Cowen L, Berger B. Sequence-based prediction of protein-protein interactions: a structure-aware interpretable deep learning model. bioRxiv. 2021; p. 2021.01.22.427866. doi:10.1101/2021.01.22.427866.

25. Bepler T, Berger B. Learning protein sequence embeddings using information from structure. In: 7th International Conference on Learning Representations. arXiv; 2019.

26. Oughtred R, Stark C, Breitkreutz BJ, Rust J, Boucher L, Chang C, et al. The BioGRID interaction database: 2019 update. Nucleic Acids Research. 2018;47(D1):gky1079-. doi:10.1093/nar/gky1079.

27. Dick K, Dehne F, Golshani A, Green JR. Positome: A Method for Improving Protein-Protein Interaction Quality and Prediction Accuracy. 2017 IEEE Conference on Computational Intelligence in Bioinformatics and Computational Biology (CIBCB). 2017; p. 1-8. doi:10.1109/cibcb.2017.8058545.

28. Bausch-Fluck D, Goldmann U, Müller S, Oostrum Mv, Müller M, Schubert OT, et al. The in silico human surfaceome. Proceedings of the National Academy of Sciences. 2018;115(46):201808790. doi:10.1073/pnas.1808790115.

29. Camacho C, Coulouris G, Avagyan V, Ma N, Papadopoulos J, Bealer K, et al. BLAST+: architecture and applications. BMC Bioinformatics. 2009;10(1):421. doi:10.1186/1471-2105-10-421. 
30. Raufman JP. Bioactive peptides from lizard venoms. Regulatory Peptides. 1996;61(1):1-18. doi:10.1016/0167-0115(96)00135-8.

31. Madeira F, Park Ym, Lee J, Buso N, Gur T, Madhusoodanan N, et al. The EMBL-EBI search and sequence analysis tools APIs in 2019. Nucleic Acids Research. 2019;47(W1):W636-W641. doi:10.1093/nar/gkz268.

32. Taguchi AT, Boyd J, Diehnelt CW, Legutki JB, Zhao ZG, Woodbury NW. Comprehensive Prediction of Molecular Recognition in a Combinatorial Chemical Space Using Machine Learning. ACS Combinatorial Science. 2020;22(10):500-508. doi:10.1021/acscombsci.0c00003. 


\section{A. Similarity-based methods}

\section{Query pair}

Windows of amino acids of

pre-determined size are extracted from the query proteins.
Interaction database

Windows in interacting

proteins with high similarity

to windows in query proteins

are identified.

\section{Scoring}

Scores of proteins whose potential interaction is supported by evidence are incremented in proportion with the strength of the evidence. The more similarities a query pair has with interacting proteins, the stronger the evidence.

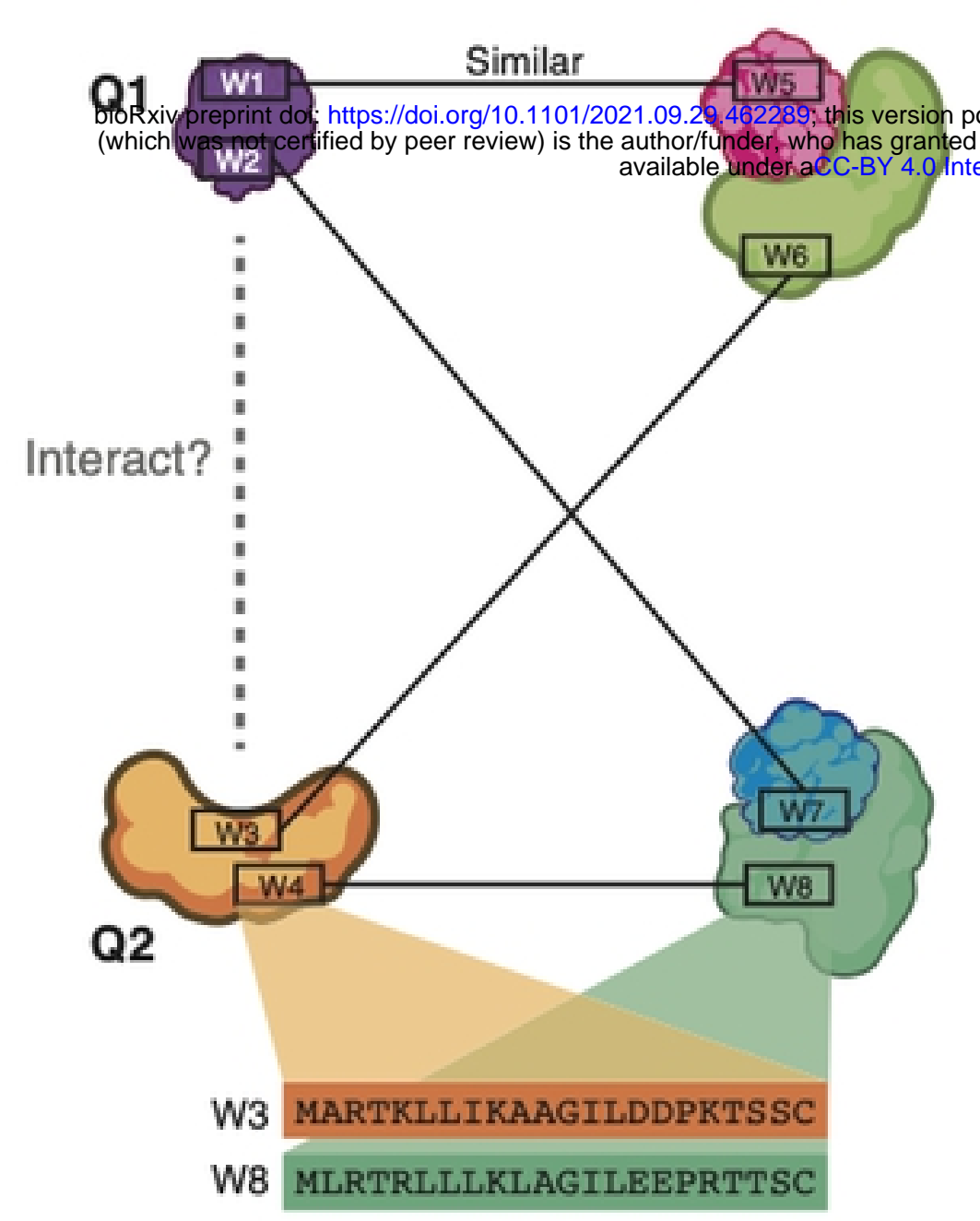

Regions similar to $\mathrm{W} 1$ and $\mathrm{W} 3$ co-occur in the interacting pair $\mathrm{P} 1$. Score of the Q1-Q2 pair is incremented accordingly
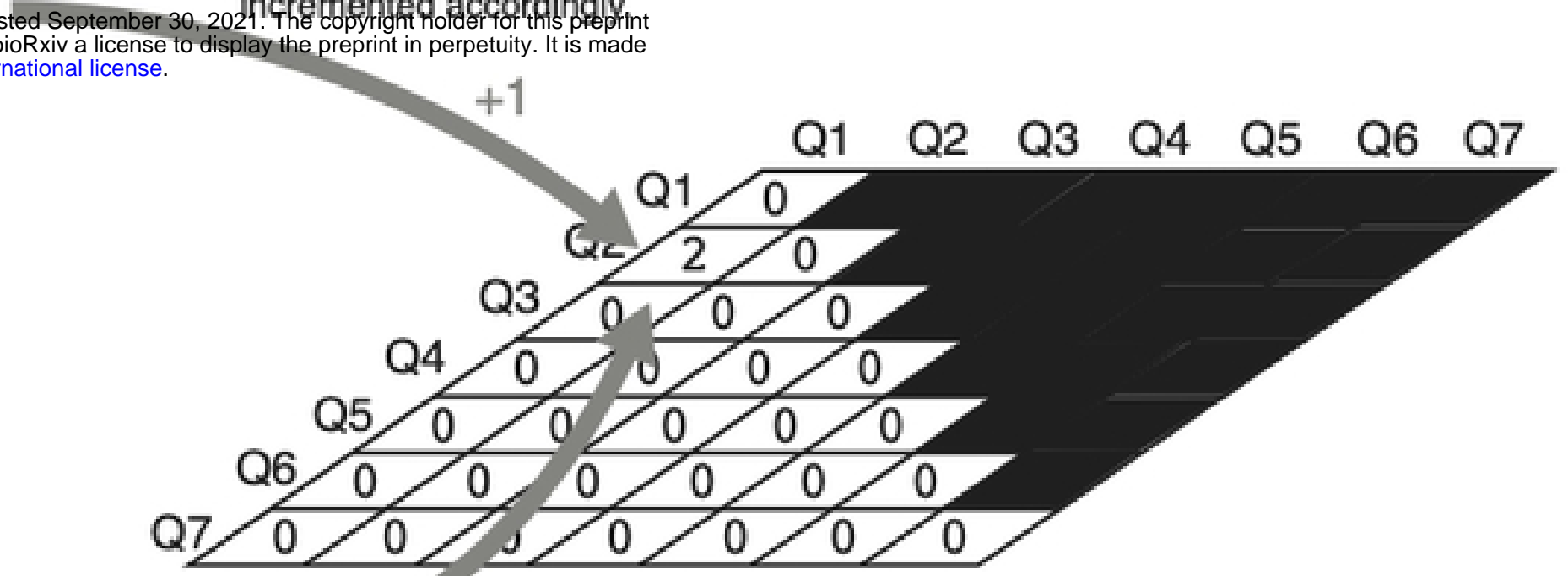
interacting pair P2. Score of the Q1-Q2 pair is incremented accordingly.

Amino acid windows similarity evaluated with a substitution matrix.

\section{B. Machine learning-based methods}

\section{Feature extraction}

Numerical features describing the protein are extracted explicitely from physicochemical properties or implicitely from amino acid composition.

\section{Classification}

A machine learning model trained on interacting and non-interacting protein pairs produces a classification from the pair's numerical representation. The model's internal parameters are a compressed representation of the interaction data.
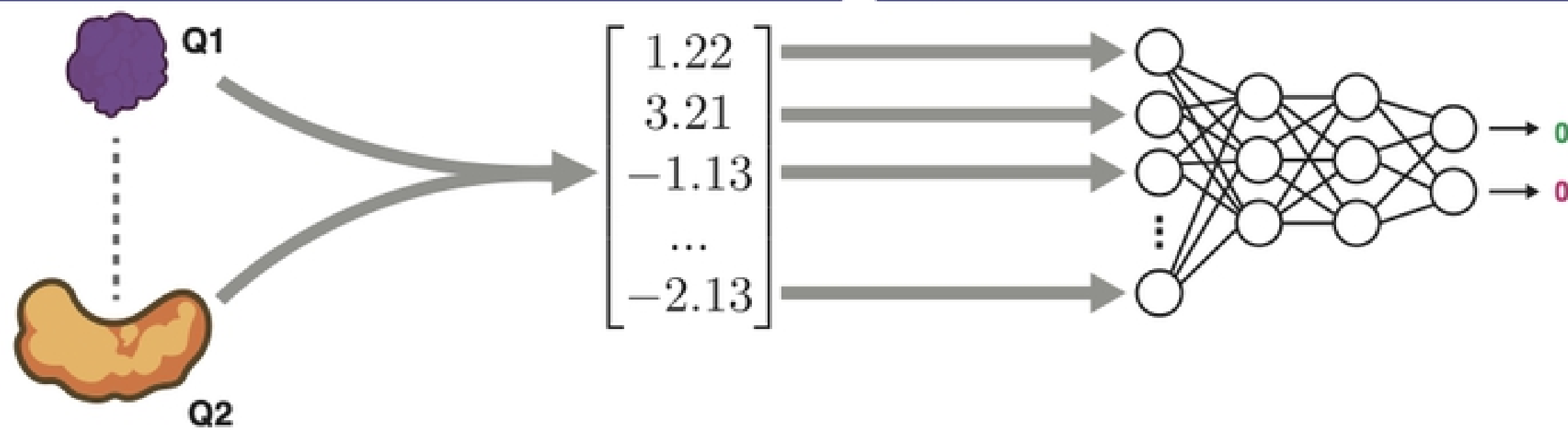

0.867 Interaction 0.133 No interaction

Figure 1 
(A) No interaction

(B) Non-specific interaction

(C) Ideal curve

PPI

score

Target protein

Rank

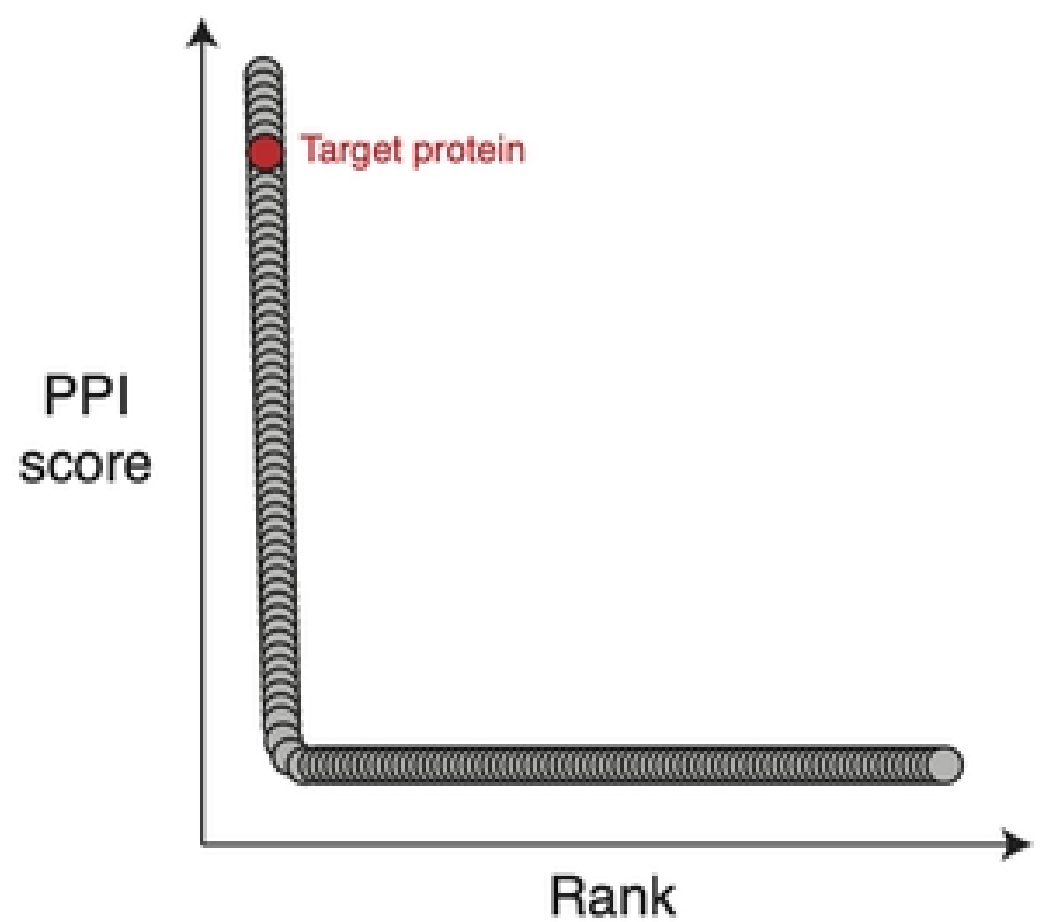

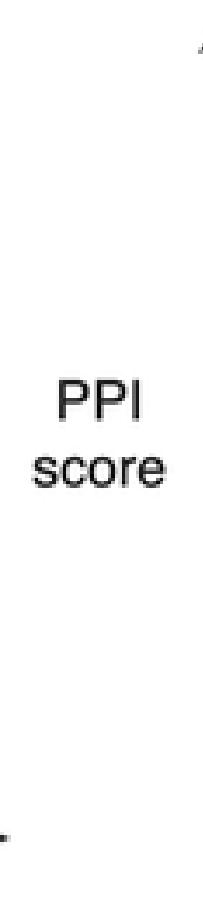

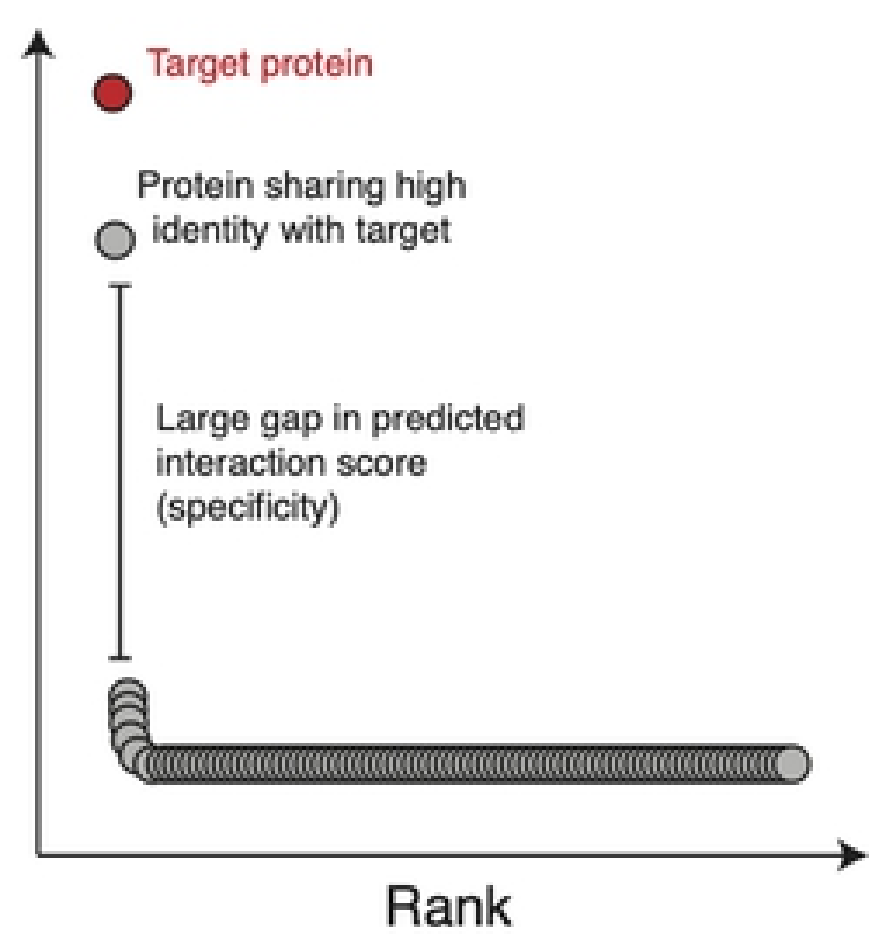

Figure 2 

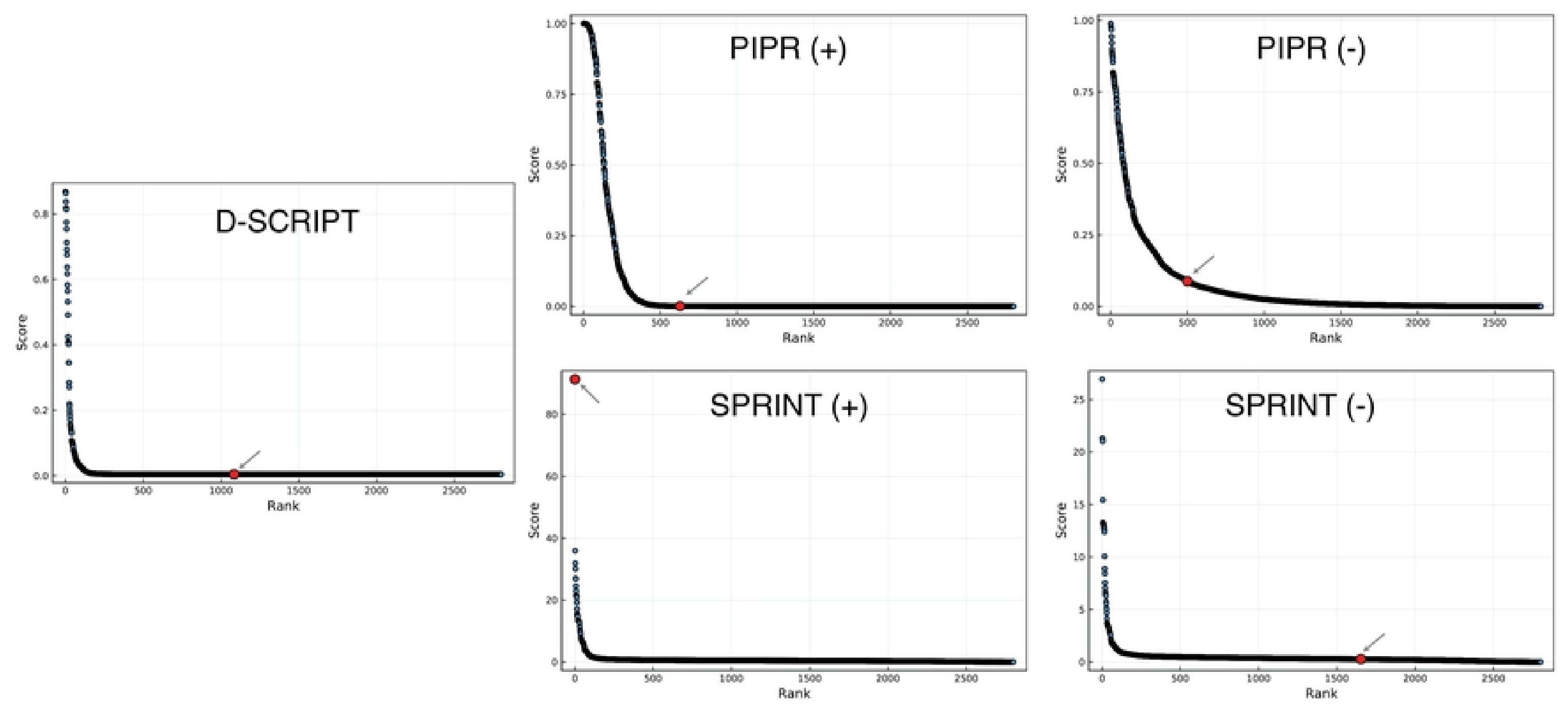

Figure 4 
CLUSTAL 0(1.2.4) multiple sequence alignment

Lixisenatide

Pro-glucagon

Lixisenatide Pro-glucagon

Lixisenatide Pro-glucagon
MKSIYFVAGLFVMLVQGSWQRSLQDTEEKSRSFSASQADPLSDPDQMNEDKRHSQGTFTS

0

60

-HGEGTFTSDLSKQMEEEAVRLFI

23

DYSKYLDSRRAQDFVQWLMNTKRNRNNIAKRHDEFERHAEGTFTSDVSSYLEGQAAKEFI

*.********:*, :* :*, : **

EWLKNGGPSSGAPPSKKKKKK-

AWLVKGRGRRDFPEEVAIVEELGRRHADGSFSDEMNTILDNLAARDFINWLIQTKITDRK

44

** :*

\section{Figure 5}




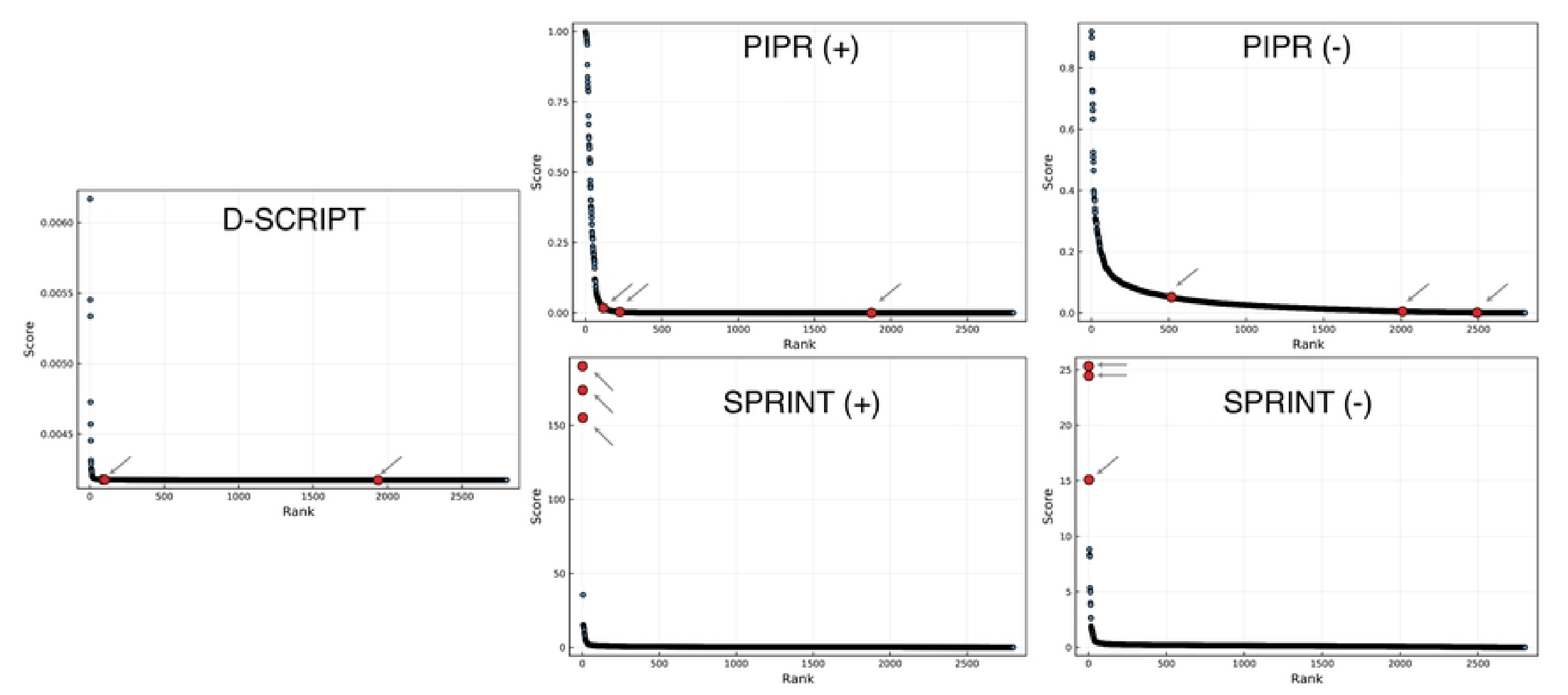

Cover Letter 